\title{
Translation, cultural adaptation and preliminary psychometric evaluation of the "Family Management Measure" among Iranian families with a child with a chronic disease
}

\author{
Masoomeh Mehmannavazan ${ }^{1}$, Meimanat Hosseini ${ }^{2}$, Jacqueline Vartanoosian ${ }^{3}$, Mahsa Matbouei ${ }^{4}$, Malihe Nasiri ${ }^{5}$, \\ Parvaneh Vasli ${ }^{2}$
}

${ }^{1}$ M.Sc. Student of Community Health Nursing, Department of Community Health Nursing, School of Nursing and Midwifery, Shahid Beheshti University of Medical Sciences, Tehran, Iran

${ }^{2}$ Ph.D. in Nursing, Assistant Professor, Department of Community Health Nursing, Faculty Member of Nursing \& Midwifery School, Shahid Beheshti University of Medical Sciences, Tehran, Iran

${ }^{3}$ M.Sc. in Medical Education, Medical Laboratory Scientist, Department of Basic Sciences, School of Nursing and Midwifery, Shahid Beheshti University of Medical Sciences, Tehran, Iran

${ }^{4}$ M.Sc. of Medical Surgical Nursing, Nursing Instructor, Department of Community Health Nursing, School of Nursing and Midwifery, Shahid Beheshti University of Medical Sciences, Tehran, Iran

${ }^{5}$ Ph.D. in Biostatistics, Assistant Professor, Department of Biostatistics, School of Nursing and Midwifery, Shahid Beheshti University of Medical Sciences, Tehran, Iran

\section{Type of article: Original}

\begin{abstract}
Background: The basis of any research is the use of valid and reliable tools for collecting information. One of the valid and reliable tools used to identify the family management in chronic childhood diseases is "FaMM" which has never been translated and validated in previous research in Iran.

Objective: The present study aimed to translate, incorporate cultural adaptation, and determine psychometric features of FaMM among Iranian families with a child with a chronic disease.

Methods: This study was conducted using the methodological research approach in Tehran, during 2016-2017. The translation of FaMM was done using "World Health Organization Translation and Cultural Adaptation Guidelines". The preliminary psychometric evaluation of the above tool was performed by determining the validity (face and content validity) and reliability (internal consistency and stability). Data analysis was performed using IBM-SPSS 22. Content validity (CV) was first examined using qualitative and quantitative methods. In the qualitative phase, experts' opinions were received in order to observe grammar, wording, item allocation and scaling. Then, quantitative CV was examined using Content Validity Ratio (CVR) and Content Validity Index (CVI; Scale-CVI /Avg, Scale-CVI/ UA). In order to calculate the internal consistency of the items, Cronbach's alpha coefficient of statements was calculated. Test-retest method was used for determining the stability of the tool, and the obtained scores were compared using ICC.

Results: The Persian version of FaMM has conceptual, semantic, idiomatic and by-item equivalence, as well as a desirable preliminary validity and reliability for assessing the management of families with children with chronic disease. The results of CVR determination indicated that all questions were larger than Lawshe's Table number (0.49). The CVI results showed that all questions had a score higher than 0.79 , and therefore were recognized as appropriate. In the Scale-CVI /Avg, the score was 0.98 and in the Scale-CVI/ UA, the score was 0.80. The Cronbach's alpha value for the scales was obtained in the range of 0.55 to 0.87 and the ICC of scales ranged from 0.71 to 0.94 .
\end{abstract}

\section{Corresponding author:}

Assistant Professor Dr. Meimanat Hosseini, Department of Community Health Nursing, School of Nursing and Midwifery, Shahid Beheshti University of Medical Sciences, Tehran. Iran.

Tel: +98.2188655366, Fax:+98.2188655384, Email: meimanathosseini@yahoo.com and m_hoseini@sbmu.ac.ir Received: September 19, 2017, Accepted: January 01, 2018, Published: June 2018

iThenticate screening: December 30, 2017, English editing: January 10, 2018, Quality control: March 12, 2018

This article has been reviewed / commented by five experts

Ethics approval: IR.SBMU.PHNM.1395.491

(C) 2018 The Authors. This is an open access article under the terms of the Creative Commons Attribution-NonCommercialNoDerivs License, which permits use and distribution in any medium, provided the original work is properly cited, the use is non-commercial and no modifications or adaptations are made. 
Conclusion: The translation and cultural adaptation process of FaMM led to the design of a tool adapted to Iranian culture which can be applied in this context, and after the implementation of the final psychometric evaluation process, comparing data on how families with a child with chronic disease are managed with other communities can be possible.

Keywords: Translation, Instrumentation, Household, Disease management, Measure

\section{Introduction}

Across the globe, the prevalence of chronic diseases in children is on the increase, extensively (1). However, due to different definitions of diseases and the absence of a national database, determining the absolute number of children with chronic disease is rather difficult $(1,2)$. Overall, it is estimated that around $18 \%$ of children, from birth to age 18 , experience some kind of chronic physical, developmental, behavioral, and emotional illnesses, and generally need more health services than others (3). In Iran, there are no accurate statistics on the prevalence of chronic diseases, but as a sample, the prevalence of asthma as the most common chronic health problem in children is 10 to $15 \%$ (2). The care required for chronic diseases is often complex, and preventing these diseases imposes unusual expectations and a significant amount of time, energy and personal resources on child and family alike (4). The family plays an important role in the care rendered to childhood chronic illnesses (4), and in different ways responds to the challenge resulting from these diseases (5). The family needs continuous management of the circumstances in order to cope with the permanent changes in child illness (5). The way the family responds to and manages the child's chronic disease will affect the outcome for the child and his/her adaptation to illness (6), as well as family function (5). Understanding how family life is managed with the presence of a child with a chronic disease is important for nurses to such families with supportive health interventions (7). In this regard, the study of family management in childhood chronic disease and identification of its patterns seems necessary (5). Nursing studies have emphasized the importance of implementing family-centered nursing interventions in order to provide a supportive environment for the patient (8). Nurses supporting and promoting the health of the child with chronic disease and his/her family, are required to adapt to the needs of the disease and the types of resources available to the family (9). Under all conditions, what is important in collecting information is the choice of research tool. Choosing the proper and right tool will lead to accurate measurement of the given variables (10). Researchers would be incapable of conducting appropriate and scientific research without relying on a proper and scientific tool. Findings from invalid tools would be questioned despite the goals, procedures, and analysis of the correct data (11). So it is stated that the basis of each research is the use of valid and reliable tools for collecting information (12). To date, many tools have been designed in the world's most widely used languages, and used in other countries. Psychometric evaluation experts of research tools constantly emphasize the proper process of translating tools, such that if attention is not given to this, the findings from the research cannot be compared with other national and international findings. In studies in which quantitative tools are used, the translation of tools into the cultural adaptation of the community studied is essential, which is not a simple process. The values reflected by the tool together with the meaning of its concepts and components, may vary from one culture to another. Therefore, the translation quality and the validity of the translated tool play an important role in validating the translated version. Thus, although the translation of the tool with the observance of cultural adaptation is a complicated process, it increases the quality of work and allows the comparison of the results in the culture of the target community with the culture of the source community (13). One of the reliable and valid tools used to identify family management in childhood chronic disease is Family Management Measure (FaMM) which reliability and validity have been reported as good in studies outside of Iran $(3,5,9)$. This tool was first designed and measured by Knafl et al. with a 2011 study on parents of children with various chronic diseases in the United States, and the results indicated the tool's desired reliability and validity. The authors stated that the use of FaMM could lead to the identification of different dimensions and aspects of the family response to childhood chronic diseases (3). Considering the necessity of research in identifying family management patterns in childhood chronic diseases as well as the positive effect of nurses' leadership in managing chronic diseases, clinical outcomes, quality of life and performance of patients and their families (14), and given that so far, no tool has been translated and measured in Iran to examine the patterns, the purpose of the present study is to translate, adapt the culture and to determine the psychometric features of FaMM among Iranian families with a child with chronic disease.

\section{Material and Methods}

This study which is a part of a larger study devoted to the translation and psychometric evaluation of FaMM was conducted with the methodological research approach in Tehran city during 2016-2017. The present study was conducted in two stages. In the first stage of the study, after obtaining permission from the designer of FaMM, the 
translation of this tool was carried out in 6 stages using "World Health Organization Translation and Cultural Adaptation Guidelines" (15).

\subsection{Translation of the instrument}

For the translation and cultural adaptation of FaMM with 53 items, first an English version of the tool was initially provided by two translators fluent in English and Persian language and culture (the first person was familiar with the research and the second person was not aware of the study objectives), separately and independently in Persian language, and all Persian equivalents were recorded for its English words and sentences. Attempt was made to avoid literal translation whilst adhering to the English text during the translation. Finally, in this stage, two complete and independent translations of FaMM were obtained. Then, a committee of 5 experts ( 3 faculty members of community health nursing and 2 experts in the field of cultural adaptation of the tool) fluent in both English and Persian languages, was formed. In this stage, the Persian translation along with the original version of the tool was provided to the experts of the aforementioned fields and they were asked to express their comments to a committee with the researchers. Finally, after the review and collaboration with these experts, the final translation with the consensus was prepared. Subsequently, for back translation, one person fluent in both English and Persian languages (different from the two translators at the first stage), who was unaware of the English version of the tool, as well as the research and its stages, translated the final Persian version of the previous stage into English language. Then, a version of the final English translation was sent to the tool designer for approval, and after receiving her comments and applying reforms, the Persian translation of FaMM was finally approved. Then, the approved version was carefully studied by 10 mothers of children with chronic disease, and vague points and inappropriate terms were reformed from their point of view. The inclusion criteria for the study were: mothers with sick children between the ages of 1 and 18 years, the diagnosis of chronic illness was carried out by a specialist, the diagnosis of the disease lasted for at least 6 months; the child has not been hospitalized in the last two months, and had no significant developmental impairment according to records or doctor's documented diagnosis. The final version at the end of this stage was edited by the experts of Persian language and literature, and then the documentation of all the above stages was done. It should be noted that the documented version included the initial version of the translation, the summary of the proposals and reforms of the Committee of Experts, the revision, a copy of the errors outlined in the pre-test and the final version of the translation.

\subsection{Validity}

The second stage of the study that was conducted with Methodological Research Approach was designed including the tool psychometric evaluation. In order to perform the psychometric evaluation of the above tool, the validity and reliability of the tool was examined. Face and content validity (CV) was used to determine its initial validity. In this regard, CV was first examined by two qualitative and quantitative methods. In the qualitative method, the opinion of 15 experts was received in the fields of instrumentation and psychometric measurement, community health nursing (family), pediatric nursing and specialist physicians in the field of chronic diseases selected on the basis of the purposive sampling in order to observing grammar, wording, item allocation and scaling. Then, quantitative $\mathrm{CV}$ was examined using Content Validity Ratio (CVR) and Content Validity Index (CVI). To determine the CVR, the panel of experts was asked to examine each statement based on a 3-part spectrum: Essential, Useful but not essential, and Not necessary. Then the responses were calculated according to the below formula:

$\mathrm{CVR}=[\mathrm{n}-(\mathrm{N} / 2)] /(\mathrm{N} / 2)$

Where " $\mathrm{n}$ " represents the number of experts who have chosen the "necessary" option and " $\mathrm{N}$ " is the total number of specialists (16). Lawshe has provided a table for determining the numerical value of CVR, which is referred to as "Minimum CVR Value" (16). The result after calculating was compared with respect to the number of experts with the criterion in the table. If the resulting number is larger than the Lawshe table, the presence of the relevant item with an acceptable level of significance $(p<0.05)$ is essential in the tool $(16)$. In the present study, the statements were retained with a CVR equal to and/or greater than 0.49 (based on the assessment of 15 experts) considered significant $(\mathrm{p}<0.05)$. The CVI was also calculated in two distinct ways, for each item of Item-CVI (I-CVI), and generally for the whole scale of Scale-CVI (S-CVI). The experts were asked to record their opinions in a 4-point spectrum of "highly relevant=4", "quite relevant=3", "somewhat relevant=2", and "not relevant=1". Then for each item, I-CVI was computed as the number of experts giving a rating of 3 or 4 , divided by the total number of experts (in accordance with the proposed formula by Waltz and Bassel (1983)). If this value is above $79 \%$, the item is appropriate. If the value is between 70 and $79 \%$, then the relevant item should be reviewed and, if the value is less than $70 \%$, the item should be removed (17). Scale-CVI/ Average (Scale-CVI/Avg) and Universal scale-CVI/ Universal (Scale-CVI/ UA) approaches were used to determine the CVI for the whole scale. In the average approach, the total of the CVI of each item was divided into the total number of items, and in the Universal 
approach, a ratio of the tool items was calculated and scored by all experts in terms of relevance 3 and 4 . In the newly designed tools, $80 \%$ and more agreement between judges is recommended (16). In the next stage, face validity was examined using two qualitative and quantitative methods. To determine the face validity qualitatively, 10 mothers of children with chronic disease were interviewed face-to-face regarding the level of difficulty, relevancy and ambiguity of words, statements and sentences of the tool (16). After reforming cases according to the mothers' opinions, at the next stage, item impact quantitative method was used to determine the face validity quantitatively. For this purpose, in order to reduce, remove inappropriate statements and determine the importance of each of the statements, the opinions of 10 mothers selected based on the purpose, were used. The items whose item impact score value was calculated equal to and/or greater than 1.50 (16) were retained for later analyses. In order to ensure the correct method of writing and rational wording of the questionnaire statements, the statements were repeatedly reviewed by the research group and their opinions were applied, and then reviewed and reformed by one expert in the field of Persian literature.

\subsection{Reliability}

For determining the reliability of the tool, two methods of internal consistency (Cronbach's alpha coefficient) and stability (interclass correlation coefficient [ICC]) were used. To calculate the internal consistency of the items, the tool was provided to 45 mothers of children with chronic disease who were selected with convenience sampling. The criteria for selecting these samples were similar to those of the previous stages of the study (pretest stage of translation and cultural adaptation and content validity). Then, the Cronbach's alpha coefficient of scales was calculated. The Cronbach's alpha value is acceptable between 0.70 and 0.80 (16). To determine the reliability of the tool stability, a test-retest method was used on 25 mothers with a two-week interval and the obtained scores were compared using the ICC. The most acceptable statistical test to calculate the stability is the ICC test. If this index is above $80 \%$, the stability rate is desired (17).

\subsection{Ethics of research}

This research Ethics Committee of Shahid Beheshti University of Medical Sciences approved the study (Ref. no.: IR.SBMU.PHNM.1395.491). In order to observe ethical considerations, informed consent was received from mothers. Also, information regarding the withdrawal of mothers from participation in the research at any time of study if they wished, observing the principle of secrecy and protecting the secrets of the subjects and adopting appropriate measures to prevent its publication, was given.

\section{Results}

The results of the Persian translation of the English version of FaMM are summarized using the "Six-part Guide to the Cultural Adaptation and Translation of the World Health Organization" as follows: In the final reforms of the tool, in the Persian translation of all statements, instead of the word "chronic condition", the word "chronic disease" was used, which has a better understanding from Iranian respondents' point of view. It should be noted that, this change was made after coordination with the main designer of the tool. Meanwhile, the word "partner" was changed to "spouse". The designer of FaMM emphasized the word "interfering" so the word "overcoming" was changed to "interfering". According to the main designer's opinion, the word "disease" was used in all statements. The purpose of using the word "organization" by the tool designer was not an organization or other organs, but that the family needs so much organization to take care of the child's disease. The word "closer" meant a sense of a strong relationship with each other due to a child's disease, which, in the eyes of the main designer, was different from the word "sincere" that was applied to the translation. At the pre-test stage of the translation guide, reform changes were made by a group of parents which are given in Table 1. For example, for the statement "my spouse and I discuss about how to manage our child's disease", respondents were uncertain about understanding the word "talk". So, the word "talk" was replaced by "dispute". In addition, illustrations were used for some of the statements. At the second stage of the research, based on the suggestions of the experts in the qualitative study of $\mathrm{CV}$, in order to clarify the statements, the word "organize" replaced the word "manage". Another change applied was the replacement of the word "child" with "kid". The results of CVR determination indicated that all questions were larger than Lawshe's table number (0.49). This was an indication that all questions were essential and important. CVI results showed that all questions had a score higher than 0.79 and therefore they were recognized as appropriate. In the Scale-CVI/Avg, score was 0.98 and in the Scale-CVI/ UA, the score was 0.80 (Table 2). The results showed the item impact for all items to be more than 1.50. Since all the statements had the index score above 1.50, with changes in the method of writing some of the statements based on the qualitative face validity, 53 items were retained. The Cronbach's alpha value for the scales was obtained in the range of 0.55 to 0.87 (Table 3). It should be noted that the Cronbach's alpha value in the case of removal of an item had no effect on the Cronbach's alpha value of the total scale, therefore, no 
statement was removed. The ICC of scales ranged from 0.71 to 0.94 (Table 4). Conducting of translation, cultural adaptation and psychometric evaluation led to the confirmation of a tool consisting of 6 scales: child's daily life (5 items), condition management ability (12 items), condition management effort (4 items), family life difficulty (14 items), parental mutuality (8 items) and view of condition impact (10 items).

Table 1. Reformed translation after a pre-test of the translation by subjects

\begin{tabular}{|l|l|}
\hline Primary translation & Final translation \\
\hline $\begin{array}{l}\text { My spouse and I discuss about how to } \\
\text { manage our child's disease }\end{array}$ & My spouse and I dispute about how to manage our child's disease \\
\hline $\begin{array}{l}\text { We know when our child needs to be a } \\
\text { child. }\end{array}$ & $\begin{array}{l}\text { We know when our child needs to be a child (for example, when he/she } \\
\text { cannot follow his/her own diet and participate in activities he/she likes). }\end{array}$ \\
\hline $\begin{array}{l}\text { Our child's disease interferes with our } \\
\text { family relationships. }\end{array}$ & $\begin{array}{l}\text { Our child's disease disturbs our family relationship (i.e. the relationship } \\
\text { between the parents and children, family and friends, and the husband and } \\
\text { wife). }\end{array}$ \\
\hline $\begin{array}{l}\text { In spite of the disease, we expect our child } \\
\text { lives away from home in the future. }\end{array}$ & $\begin{array}{l}\text { Despite the disease, we expect our child can have an independent life in the } \\
\text { future. }\end{array}$ \\
\hline
\end{tabular}

Table 2. CVR and CVI of the FaMM

\begin{tabular}{|c|c|c|c|c|c|}
\hline \multirow[t]{2}{*}{ No. } & \multirow[t]{2}{*}{ Items of FaMM } & \multirow[t]{2}{*}{ CVR } & \multicolumn{3}{|l|}{ CVI } \\
\hline & & & I-CVI & \begin{tabular}{|l|} 
S-CVI \\
/ UA
\end{tabular} & $\begin{array}{l}\text { S-CVI } \\
\text { / Avg }\end{array}$ \\
\hline 1 & Our child's everyday life is similar to that of other children of his/ her age. & 0.73 & 0.93 & & \\
\hline 2 & Our child's disease interferes with family relationships. & 0.86 & 0.93 & & \\
\hline 3 & Our child needs frequent clinical checks and observations. & 0.86 & 1 & & \\
\hline 4 & In the future, we expect our child to take care of his disease. & 0.86 & 1 & & \\
\hline 5 & Our child, because of his/her disease, has less enjoyment from life. & 0.6 & 1 & & \\
\hline 6 & We are often exhausted from caring for our sick child. & 0.73 & 1 & & \\
\hline 7 & Our child's disease is like a roller coaster with lots of ups and downs. & 0.86 & 1 & & \\
\hline 8 & Our child's disease is the most important thing in our family. & 0.86 & 1 & & \\
\hline 9 & It's very difficult for us to care for our child's disease. & 0.86 & 1 & & \\
\hline 10 & Our child, despite his/her disease, participates in activities that he/she wishes. & 0.86 & 1 & & \\
\hline 11 & Due to disease, we are concerned about the future of our child. & 0.86 & 0.93 & & \\
\hline 12 & Managing our child's disease is not time consuming. & 0.86 & 0.93 & & \\
\hline 13 & We have some clear ideas about how we help our child to live with his/her disease. & 1 & 1 & & \\
\hline 14 & Despite the disease, we expect our child to live away from home in the future. & 0.73 & 1 & & \\
\hline 15 & We have enough money to manage our child's disease. & 0.86 & 1 & & \\
\hline 16 & Due to disease, our child is different from other children his/her age. & 0.73 & 1 & & \\
\hline 17 & Knowing the fact that, when designing, priority for our child's disease in the family is difficult. & 0.86 & 0.93 & & \\
\hline 18 & We look forward to a happy future for our child. & 1 & 1 & & \\
\hline 19 & $\begin{array}{l}\text { When something unexpected occurs in relation to the disease of our child, we usually know how } \\
\text { to handle it. }\end{array}$ & 0.86 & 1 & & \\
\hline 20 & Our child's relationship with his/her friends is different due to his/her disease. & 0.73 & 0.93 & & \\
\hline 21 & We expect to dedicate less time for our child's disease in future. & 0.86 & 1 & & \\
\hline 22 & A condition similar to that of our sick child, makes family life very difficult. & & & & \\
\hline 23 & Disease of our child rarely interacts with family activities. & 0.86 & 1 & & \\
\hline 24 & Our sick child requires frequent hospitalization. & 0.86 & 1 & & \\
\hline 25 & We feel we are doing a good job of taking care of our child's disease. & 1 & 1 & & \\
\hline 26 & Children who have the same disease as our child, live a normal life span. & 1 & 1 & & \\
\hline 27 & It is often difficult to know whether we need to be more protective of our child. & 0.73 & 0.86 & & \\
\hline 28 & We often don't know what we should do to take care of our child's disease. & 0.86 & 1 & & \\
\hline 29 & It will be more difficult in the future to take care of our child's disease. & 0.86 & 1 & & \\
\hline 30 & We are constantly thinking about our child's disease. & 0.60 & 1 & & \\
\hline 31 & It seems that our child's disease controls our family life. & 0.86 & 1 & & \\
\hline 32 & There are more serious diseases than our child's disease. & 0.86 & 1 & & \\
\hline 33 & It is difficult to get someone else to help us with our sick child. & 0.73 & 0.93 & & \\
\hline 34 & We have been unable to develop a routine of taking care of our sick child. & 0.86 & 1 & & \\
\hline 35 & To manage our child's condition, a lot of organization were needed. & 0.86 & 0.93 & & \\
\hline
\end{tabular}




\begin{tabular}{|l|l|l|l|l|}
\hline 36 & Sometimes we have doubts, on how to create a balance between family life and disease. & 0.86 & 1 \\
\hline 37 & Knowing what to expect in the future of my child's disease, is difficult. & 0.86 & 1 \\
\hline 38 & Although our child is sick, we have a normal family life. & 0.86 & 1 \\
\hline 39 & Our child would have achieved better in school if he/she was not sick. & 0.73 & 1 \\
\hline 40 & We are confident that we can take care of our sick child. & 0.60 & 0.86 \\
\hline 41 & We have objectives and ideas in our mind that help us to manage our child's disease. & 1 & 1 & \\
\hline 42 & It is hard to fit the treatments needed for our sick child into our daily life. & 1 & 1 \\
\hline 43 & Coping with our child's disease makes family life difficult. & 0.86 & 1 \\
\hline 44 & We know when our child needs to behave as a child. & 1 & 1 \\
\hline 45 & It is difficult to have a normal life, while having a sick child. & 1 & 1 \\
\hline 46 & We are a closer family through coping with our child's disease. & 0.86 & 1 \\
\hline 47 & My spouse and I have different opinions about how serious our child's disease is. & 1 & 1 \\
\hline 48 & I am satisfied how my spouse and I handle our child's disease. & 1 & 1 \\
\hline 49 & My spouse and I talk to each other about how to manage our child's disease. & 1 & 1 \\
\hline 50 & $\begin{array}{l}\text { My spouse and I, consult with each other before making any decision about taking care of our } \\
\text { child. }\end{array}$ & 1 & 1 \\
\hline 51 & My spouse and I have common ideas on how to raise our child. & & \\
\hline 52 & I am not satisfied with the way that my wife and I assign duties to care for our sick child. & 0.73 & 1 \\
\hline 53 & My spouse and I support each other while we take care of our sick child. & 0.86 & 1 \\
\hline & Child's Daily Life & 0.86 & 1 & \\
\hline & Condition Management Ability & & \\
\hline & Parental Mutuality & & \\
\hline Condition Management Effort & & \\
\hline Family Life Difficulty & & \\
\hline View of Condition Impact & & \\
\hline Total & & \\
\hline
\end{tabular}

FaMM: "Family Management Measure"

Table 3. The Cronbach's alpha value for the scales of FaMM

\begin{tabular}{|l|l|l|}
\hline Scales & Item no. & Cronbach's alpha \\
\hline Child's Daily Life & 5 & 0.87 \\
\hline Condition Management Ability & 12 & 0.66 \\
\hline Parental Mutuality & 8 & 0.87 \\
\hline Condition Management Effort & 4 & 0.55 \\
\hline Family Life Difficulty & 14 & 0.86 \\
\hline View of Condition Impact & 10 & 0.74 \\
\hline
\end{tabular}

FaMM: "Family Management Measure"

Table 4. Stability reliability of FaMM

\begin{tabular}{|l|l|l|}
\hline Scales & ICC consistency & ICC agreement \\
\hline Child's Daily Life & $0.92(0.83-0.96)$ & $0.92(0.83-0.96)$ \\
\hline Condition Management Ability & $0.71(0.35-0.87)$ & $0.71(0.36-0.87)$ \\
\hline Parental Mutuality & $0.92(0.82-0.96)$ & $0.92(0.82-0.96)$ \\
\hline Condition Management Effort & $0.90(0.79-0.95)$ & $0.90(0.79-0.95)$ \\
\hline Family Life Difficulty & $0.87(0.71-0.94)$ & $0.87(0.71-0.94)$ \\
\hline View of Condition Impact & $0.94(0.87-0.97)$ & $0.94(0.85-0.97)$ \\
\hline
\end{tabular}

FaMM: "Family Management Measure"; ICC: Interclass Correlation Coefficient

\section{Discussion}

The results of the study showed that the translation and cultural adaptation of FaMM is acceptable. Among the 53 tool statements, according to the opinion of the main tool designer and the committee of experts, all the statements that included the word "chronic condition" were changed into the statements with the word "chronic disease" that could be better understood. Similar to the present study, Ichikawa et al. also used the word "chronic disease" in their translation (9). Most parents were confused about the concept of "living away from home" in the statement "in spite of the disease we expect in the future that our child lives away from home". Comparing the finding of the present study on the translation with other similar studies, including the research by Ichikawa et al. in Brazil (9) and Hutton 
et al. in Australia (6), such a conclusion cannot be drawn which might be due to the close proximity of culture of the two countries and the country of the main study related to the tool design (United States). In the culture of the mentioned countries, the separation of children from the family is common in adolescence. But in Iranian culture, this seems to be an unfamiliar concept. The word "partner" was renamed as "spouse" in the statements that included this word. Because the concept of "partner" was ambiguous from the point of view of Iranian respondents, and on the other hand, the word is not applicable in Iranian culture. At the second stage of the research, in determining the CV qualitatively, for much more clarity, the word "manage" was changed to "organize", because the word "manage" from the point of view of experts, was more of the concept of "organizational management". Knafl et al. described family management as efforts put in by family members in order to integrate subsequent expectations of the disease and the needs of a child with chronic disease within a family's everyday life (5). Comparing the findings of the present study with other similar studies, including the research by Ichikawa et al. (9) and Hutton et al. (6), such a result was not found, which is likely due to the close proximity of the culture of the two countries and the main study country related to the tool design. At the quantitative CV stage, the score of all questions in the CVR stage was larger than the Lawshe Table number, which indicated the necessity and importance of the tool questions. In all the studies where FaMM was used (3, 5, 6, 9 and 18), CVR had not been reported, and this study is the first to address such an issue. In the present study, the Scale-CVI/ Average was 0.98 and scale-CVI/ Universal was 0.80, which is desired. In none of the studies on FaMM validation, has no report of the CVI been presented $(3,5,6,9,18)$. The results of face validity were desired using a qualitative and quantitative approach. In none of the studies conducted on the mentioned tool abroad, examining the face validity quantitatively and calculating the "item impact" have not been reported $(3,5,6,9,18)$, and it seems that in all, only the qualitative face validity has been used. In the present study, the results of internal consistency for all scales, except for the scale of condition management ability and condition management effort, were above 0.7 , indicating the internal stability of the tool. The Cronbach's alpha coefficient in the scales of condition management effort and the condition management ability was 0.55 and 0.66 , respectively, indicating that the internal consistency of the two statements is intermediate. In a 2014 study, Ichikawa et al. reported that Cronbach's alpha value for the scales of Child's daily life, Condition management ability, Condition management effort, Parental mutuality, Family life difficulty, and View of condition impact were $0.79,0.54,0.36,0.79,0.78$ and 0.42 , respectively (9). These authors considered the Cronbach's alpha value of more than 0.40 to be acceptable. Therefore, all scales, except for the scale of the Condition management effort, had an acceptable internal consistency. They stated that the statements of Condition management effort scale, due to the low internal consistency score, indicate that this scale measures different features and requires a review at the next stage of the study. A 2013 study by Kim and Im indicated reliable internal consistency of a Korean version of FaMM with Cronbach's alpha of 0.79, 0.74, 0.69, 0.91, 0.71 and 0.89 on the scales of Child's daily life, Condition management ability, Condition management effort, Family life difficulty, View of condition impact and Parental mutuality (18). In a 2011 study conducted by Knafl et al., the Cronbach's alpha value for all scales was obtained in the range from 0.72 to 0.91 (3). The present study is the first study in which attempt has been made to examine the internal consistency of FaMM statements after determining the face and content validity. Since the internal consistency value of the scales of the Condition management effort and Condition management ability was in the mean range, at the final stage of the study, these scales will be further assessed. Also, in the present study, the ICC coefficient for the whole scales of the tool was in the range of 0.71 to 0.94 which indicates the high stability of the tool. In the main study, also, ICC was within the same range (3). This research was conducted on a sample of mothers of children with chronic disease. Since, in most cases, only the mother with the child referred to the centers under the study or the mother often tended to participate in the study, the samples only consisted of the mothers. Therefore, it was not possible to examine the father's perception of children with chronic disease on the family management.

\section{Conclusions}

According to the results and purpose of the study, the Persian version of FaMM called "Family Management Measure" has conceptual, semantic, idiomatic and by-item equivalence for assessing the management of families having a child with a chronic disease. The translation and cultural adaptation process of FaMM has resulted in the preparation of a tool adapted to Iranian culture that can be applied in this context, and makes data comparison with other communities possible. The preliminary psychometric results of the tool also indicated good face and content validity, and the reliability of the tool. After the stage of the final psychometric process (determining the construct validity and the final internal consistency of the tool), which is the final stage in the research, this tool will be able to assess the way of management of families having a child with a chronic disease. 


\section{Acknowledgments:}

This paper is part of a nursing MS dissertation, and was approved by the Ethics Committee of Shahid Beheshti University of Medical Sciences (IR. SBMU. PHNM.1395.401). The authors would like to express their gratitude to Shahid Beheshti University of Medical Sciences for their financial support and to all the participants for the honest expression of their ideas, and also to the Thalassemia Association manager, and managers of the clinics affiliated to Shahid Beheshti and Tehran University of Medical Sciences who granted permission to use their facilities.

\section{Conflict of Interest:}

There is no conflict of interest to be declared.

\section{Authors' contributions:}

Study concept and design: Masoomeh Mehmannavazan, Meimanat Hosseini, Jacqueline Vartanoosian, Mahsa Matbouei, Malihe Nasiri, Parvaneh Vasli; Acquisition of data: Masoomeh Mehmannavazan; Analysis and interpretation of data: Masoomeh Mehmannavazan, Meimanat Hosseini; Drafting of the manuscript: Masoomeh Mehmannavazan; Critical revision of the manuscript for important intellectual content: Meimanat Hosseini, Jacqueline Vartanoosian, Mahsa Matbouei; Administrative, technical and material support: Meimanat Hosseini; Study supervision: Meimanat Hosseini.

\section{References:}

1) Beacham BL. Perspectives of Family Management from School-Aged Children with Chronic Health Conditions: Through the Eyes of the Children. University of Pennsylvania. 2013: 140.

2) Nikfarid L, Eezadi H, Shakoori M. Coping Behaviors of Mothers of Chronically Ill Children. IJN. 2012; 24(74): 53-62.

3) Knafl KA, Deatrick JA, Gallo A, Dixon J, Grey M, Knafl G, et al. Assessment of the Psychometric Properties of the Family Management Measure. J Pediatr Psychol. 2011; 36(5): 495-505. doi: 10.1093/jpepsy/jsp034. PMID: 19451173, PMCID: PMC3131701.

4) Zhang Y, Wei M, Shen N, Zhang Y. Identifying Factors Related to Family Management During the Coping Process of Families with Childhood Chronic Conditions. A Multi-Site Study. J Pediatr Nurs. 2015; 30(1): 160-73. doi: 10.1016/j.pedn.2014.10.002. PMID: 25450446.

5) Knafl KA, Deatrick J, Knafl G, Gallo A, Grey M, Dixon J. Patterns of Family Management of Childhood Chronic Conditions and Their Relationship to Child and Family Functioning. J Pediatr Nurs. 2013; 28(6): 523-35. doi: 10.1016/j.pedn.2013.03.006. PMID: 23602651. PMCID: PMC4316683.

6) Hutton A, Munt R, Aylmer C, Deatrick J. Using the Family Management Measure in Australia. Neonatal Paediatric\& Child Health Nursing. 2012; 15(2): 17-25.

7) Kim D, Im Y. The Influence of Family Management Style on Psychosocial Problems of Childhood Cancer Survivors in Korea. Eur J Oncol Nurs. 2015; 19(2): 107-12. doi: 10.1016/j.ejon.2014.10.010. PMID: 25466826.

8) Modanloo Sh, Rohani C, Farahani Shirin Abadi A, Pourhossein gholi A. Assessment of Family Function among Parents of Children with Cancer. IJNR. 2015; 10(1): 56-65.

9) Ichikawa C, Bousso R, Misko M, Mendes-Castillo A, Chiaradia Mendes-Castillo A. Cultural Adaptation of The Family Management Measure among Families of Children and Adolescents with Chronic Diseases. Rev Lat Am Enfermagem. 2014; 22(1): 115-22. doi: 10.1590/0104-1169.2978.2379. PMID: 24553711, PMCID: PMC4292687.

10) Mahmoudi S. Translation and Psychometric of "Copenhagen Burnout Inventory": Shahid Beheshti University of Medical Sciences and Health Services. 2014.

11) Polit D, Beck CT. Nursing Research: Generating and Assessing Evidence for Nursing Practice. Philadelphia: Lippincott Williams \& Wilkins; 2012.

12) Yaghmaei F. Measuring Behavior in Research by Valid and Reliable Instruments. Tehran: Shahid Beheshti University of Medical Sciences and Health Services; 2005.

13) Pashandi SH, Khaghanizadeh M, Ebadi A. Review of translation and cultural adaptation process of questionnaires. ESMS. 2009; 2(3): 117-20.

14) Wilson PM, Brooks F, Procter S, Kendall S. The Nursing Contribution to Chronic Disease Management: A Case of Public Expectation? Qualitative Findings from a Multiple Case Study Design in England and Wales. Int J Nurs Stud. 2012; 49(1): 2-14. doi: 10.1016/j.ijnurstu.2011.10.023. PMID: 22093577.

15) World Health Organization. Translation and Cultural Adaptation Guideline. 2013. Available from: http://www.who.org. 
16) Ebadi A, Zarshenas L, Rakhshan M, Zareiyan A, Sharifnia SH, Mojahedi M. Principles of scale development in health science. Tehran: Jame-e-Negar; 2017.

17) Polit D, Beck CT. Essentials of nursing research, methods, appraisals and utilization. Sixth ed. Philadelphia: Lippincott; 2015.

18) Kim DH, Im YJ. Validity and reliability of Korean version of the Family Management Measure (Korean FaMM) for families with children having chronic illness. J Korean Acad Nurs. 2013; 43(1): 123-32. doi: 10.4040/jkan.2013.43.1.123. PMID: 23563075. 